\title{
Limitantes a la inversión productiva como obstáculo al desarrollo. El caso de la Argentina
}

Constraints to productive investment as obstacles to development. The case of Argentina

\section{Marta Bekerman}

Directora del Centro de Estudios de la Estructura Económica (CENES)

Facultad de Ciencias Económicas, UBA marbekerman@gmail.com

\section{Anabel González Chiara}

Investigadora del Centro de Estudios de la Estructura Económica (CENES)

Facultad de Ciencias Económicas, UBA anabel.gonzalez@fce.uba.ar

\section{Resumen}

El trabajo comienza por enfatizar el rol de la inversión productiva, no sólo para posibilitar la acumulación de factores, sino también como elemento central para lograr la generación y absorción de tecnología y lograr una transformación estructural que permita diversificar las exportaciones y reducir su concentración en productos primarios. A partir de allí intenta analizar cuáles son los obstáculos que limitan la expansión de las inversiones en el marco de las economías latinoamericanas, con particular referencia a la Argentina. Con tal fin toma en cuenta factores vinculados, por un lado, a las características de la estructura productiva y, por el otro, a aquellos derivados de políticas públicas inefectivas. En ese contexto se presenta a las políticas productivas como un instrumento clave para sortear los obstáculos que limitan la inversión, y se plantea un conjunto de instrumentos vinculados a la intervención de los mercados o a la provisión de bienes públicos tomando en cuenta su interacción tanto a nivel horizontal como vertical.

Fecha de recepción:

6.2.18

Palabras clave: política económica - desarrollo - inversiones - provisión de bienes públicos - Argentina.

Fecha de aceptación:

23.2.18 


\begin{abstract}
This paper remarks the role of investment not only to allow the accumulation of productive factors, but also to promote the generation and absorption of technology to make possible a process of export diversification. Then, an analysis of which are the obstacles for the expansion of investments in the Latin American economies, with particular reference to the Argentina, is undertaken. It takes into account factors linked both to the characteristics or the productive structure and to the implementation of ineffective public policies. In that context productive policies are presented us a crucial instrument to counteract those obstacles and a set of instruments linked to market interventions and to the provision of public goods are introduced taking into account their interaction at the horizontal and vertical level
\end{abstract}

Key-words: economic policy - economic development investments - provision of public goods - Argentine.

\title{
Introducción
}

El actual proceso de estancamiento en el precio de los commodities, la lenta reactivación de las economías del norte, la desaceleración de la economía china (uno de los principales socios comerciales de la región), son factores que, entre otros, establecen límites al crecimiento de los países de América del Sur. La región presenta ventajas comparativas en recursos naturales, fuertemente dependientes de los precios internacionales, mientras que son pocos los avances logrados en la diversificación de la matriz productiva y exportadora con el fin de reducir la dependencia externa y lograr un crecimiento sustentable a partir del desarrollo de ventajas competitivas dinámicas.

La situación de los países emergentes que no pueden recurrir a mejorar su competitividad en base a bajos salarios ha sido calificada por distintos autores como la "trampa de los ingresos medios". Se trata de países que enfrentan el reto de adoptar las reformas necesarias para incrementar la productividad vía inversiones en capital e innovaciones (Foxley y Sossdorf 2011). Aquellos que han logrado salir de la trampa, como es el caso de Corea del Sur, han basado su crecimiento en la "economía creativa" a partir de una convergencia de la ciencia y la tecnología con la industria y con una gran apuesta a la educación, a la innovación y a la I+D. A partir de ello, cabe preguntarse por qué los 
países de la región no han logrado implementar reformas estructurales que incentiven la inversión tanto en capital físico como humano, de modo de poder asegurar un proceso de crecimiento sustentable y equitativo.

En ese contexto, este trabajo apuntará a evaluar los limitantes que actualmente enfrentan las economías latinoamericanas, con particular referencia a la argentina, para desarrollar un proceso de transformación en favor de las actividades de mayor productividad y sofisticación tecnológica, así como para lograr un desplazamiento del empleo hacia ese tipo de actividades. Para ello se comenzará analizando el rol de la inversión productiva y la innovación, como ejes centrales para lograr dicha transformación (Sección I). En la Sección II se presentan las limitantes derivadas de las características que presenta la estructura productiva y los derivados de las políticas públicas. Luego (Sección III) se delinean elementos de políticas que puedan promover las transformaciones señaladas, para finalmente presentar algunas conclusiones.

\section{Inversión Productiva: variable central del desarrollo a largo plazo. La situación en la Argentina}

Ante el avance en el proceso de globalización, la innovación y el avance tecnológico cobran cada vez más relevancia para insertarse en el mercado mundial a partir del desarrollo de ventajas competitivas dinámicas que permitan avanzar más allá de las exportaciones de materias primas sujetas, en general, a factores exógenos como el clima y los precios internacionales. Pero todo proceso de desarrollo dinámico requiere un sendero estable de inversiones productivas que haga posible la acumulación de factores y la generación y absorción de tecnología. Aquí hay que resaltar que, además de la inversión en capital físico (maquinaria, infraestructura, transporte, herramientas, etc.), es imprescindible la inversión en capital humano ligada a la generación y acumulación de conocimiento.

La falta de inversión no sólo puede limitar los procesos innovadores; en el corto plazo puede ser uno de los factores que contribuyan a la suba en el nivel general de precios. Es que, en una situación de expansión de la demanda agregada, si no se produce una adaptación de la oferta disponible ante la falta de inversión (FBCF), ${ }^{1}$ los precios pueden incrementarse generando procesos inflacionarios. Es decir que pueden plantearse límites a la posibilidad de afrontar incrementos en los niveles de consumo. Esta situación pudo evidenciarse en Argentina durante los últimos años a partir de las diferentes tendencias que muestran el comportamiento del consumo y de la inversión. Como puede observarse en el Gráfico N¹, desde el año 2011 la inversión muestra un claro proceso de estancamiento en un contexto de fuerte crecimiento en el nivel del consumo. Es así que, a lo largo de la década, la participación del consumo privado dentro del PIB revela una fuerte tendencia positiva, mientras que la inversión creció en un primer período para luego mostrar un comportamiento levemente decreciente. En este caso podría señalarse 
que la falta de inversiones limitó el incremento en la oferta de bienes y servicios, acorde al crecimiento del consumo privado; lo que contribuyó a motorizar un aumento en el nivel general de precios.

\section{Gráfico $\mathbf{N}^{\circ} 1$}

Oferta y Demanda globales - como \% del PBI -

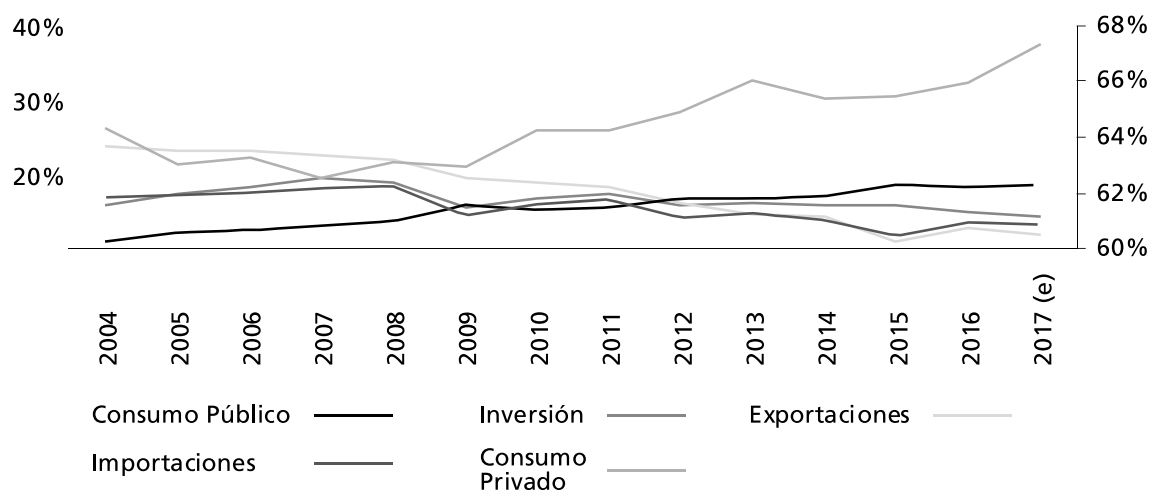

Elaboración propia en base a datos de INDEC

Más allá del corto plazo, el déficit de inversiones e innovación puede acentuar una situación de dependencia tecnológica que contribuya a consolidar en el tiempo el déficit comercial en distintos sectores, como es el caso de insumos y bienes de capital. En la Argentina, mientras que hacia comienzos de los noventa la incidencia de maquinaria y equipos importados en el total invertido rondaba el 40\%, hacia el período 2010-2012 dicha participación había superado el 60\% (Castells et al. 2014). Según cálculos realizados por algunos autores, para garantizar que Argentina logre un crecimiento sostenible -en torno al 5\%-, se necesita una tasa de inversión del 25\% del PIB (Coremberg 2015); esto contrasta con niveles actuales de inversión, los que están en torno al 18\%, según se observa en el Gráfico 1.

Esta situación se registra también en otros países de la región. Los niveles de inversión en Sudamérica han disminuido significativamente con el transcurso del tiempo, encontrándose actualmente cinco puntos porcentuales debajo de promedio mundial (WEO, 2016). La financiación de la formación bruta de capital depende fundamentalmente del nivel de ahorro, tanto interno como externo; no obstante, este último puede llegar a afectar el equilibrio de balanza de pagos. Asimismo, la tasa de ahorro interno muestra una tendencia descendente, por lo que la región continúa siendo una importadora neta de capital.

Por otro lado, en el Gráfico 2 se puede observar que en Argentina la participación del ahorro en el PBI se encuentra muy por debajo de otros países que han logrado un 
sendero de crecimiento sostenido, como India y Japón. En este contexto cabe resaltar la importancia que tienen tanto el nivel de ahorro como su eficiente canalización, las posibilidades de inversión. En contextos inflacionarios que presentan tasas de interés negativas en términos reales, se producen desincentivos al ahorro en el mercado interno, lo que tiende a alentar la fuga de capitales. Pero los bajos niveles de ahorro e inversión pueden responder asimismo a causas más profundas que serán analizadas a continuación.

\section{Gráfico $\mathbf{N}^{\circ} 2$}

Ahorro nacional bruto - como \% del PBI -

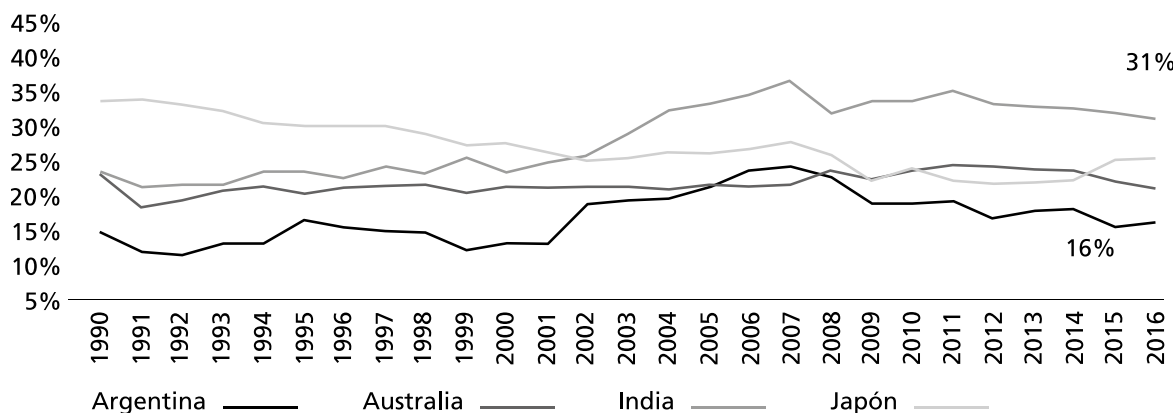

Elaboración propia en base a datos WEO 05-2016

\section{Factores Limitantes de la Inversión Productiva y de las Innovaciones.}

En esta sección se analizará el conjunto de factores que puede actuar como limitante a la expansión de las innovaciones e inversiones, especialmente aquellas orientadas hacia el largo plazo. Los mismos serán clasificados como derivados de dos factores fundamentales: a) aquellos resultantes de las características que presenta la estructura económica y b) los derivados de políticas económicas ineficientes. Es indudable que ciertas características que presenta la estructura económica pueden condicionar o limitar las posibilidades de generar nuevas estrategias de transformación productiva. Sin embargo, esas características pueden ser gradualmente modificadas a partir de la implementación de políticas públicas bien direccionadas. A continuación, se abordarán ambos factores y la relación entre los mismos.

\section{A. Factores derivados de la Estructura Económica}

\section{II.A.1 Grado de concentración y extranjerización de la estructura productiva}

La Argentina presenta una fuerte concentración y extranjerización de su estructura industrial. Al analizar el núcleo duro de empresas líderes en el país, se observa una 
hegemonía del capital extranjero, situación que se ha consolidado durante la posconvertibilidad. En efecto, la cantidad de empresas extranjeras dentro la elite empresarial, constituida por las doscientas empresas más grandes del país, pasó de 99 en 2003 a 105 en 2012, alcanzando en este último año al 52\% de los montos totales facturados por dicha cúpula empresarial. ${ }^{2}$ Este aumento se dio especialmente en los sectores agroindustrial y de minería, así como en otros sectores vinculados a actividades comerciales y de servicios (Bekerman y Vázquez, 2016). ${ }^{3}$

\section{Cuadro $\mathrm{N}^{\mathbf{0}} \mathbf{1}$}

Participación de las Empresas Extranjeras en los montos de ventas de la elite empresarial por sector 2003 y 2012

\begin{tabular}{|l|c|c|}
\hline Rama & $\mathbf{2 0 1 2}$ & $\mathbf{2 0 0 3}$ \\
\hline Total de Sectores productores de bienes & $52 \%$ & $65 \%$ \\
\hline Otros & $44 \%$ & $47 \%$ \\
\hline Industria Automotriz & $98 \%$ & $100 \%$ \\
\hline Maquinaria y Equipo & $26 \%$ & $88 \%$ \\
\hline Metales comunes y elaboración & $9 \%$ & $0 \%$ \\
\hline Minerales no metálicos & $100 \%$ & $15 \%$ \\
\hline Químicos, caucho y plástico & $86 \%$ & $83 \%$ \\
\hline Madera, papel, edición e impresión & $57 \%$ & $39 \%$ \\
\hline Textiles y calzado & s.d. & $50 \%$ \\
\hline Minería & $100 \%$ & $100 \%$ \\
\hline Petróleo y Gas & $34 \%$ & $96 \%$ \\
\hline Actividades agroalimentarias & $58 \%$ & $58 \%$ \\
\hline
\end{tabular}

Fuente: Bekerman y Vázquez (2016) 


\section{Gráfico $\mathbf{N}^{\circ} 3$}

Grado de Concentración - base 200 empresas -

\section{- 50 empresas más grandes $\quad$ Resto de las empresas}
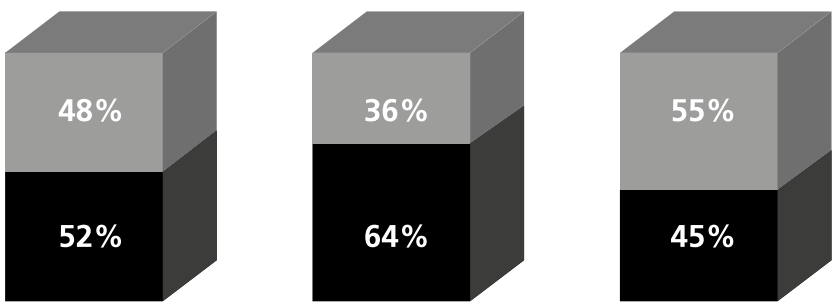

Valor Agregado

Utilidad

Puestos de trabajo asalariado

Fuente: elaboración propia en base a datos del INDEC - 2013 -

\section{Gráfico $\mathbf{N}^{\circ} 4$}

Grado de Extranjerización - base 200 empresas -

\section{- Capital extranjero (\%) ～Capital nacional (\%)}

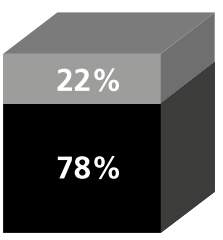

Valor Agregado

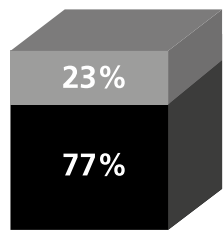

Utilidad

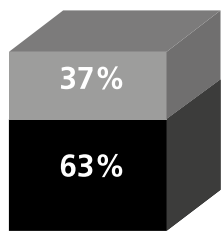

Puestos de trabajo asalariado

La elevada participación del capital extranjero dentro de la estructura productiva argentina puede generar restricciones a la inversión e innovación endógena. Esto puede originarse por diversas razones, entre las que se enumeran:

- Balanza de Pagos: desde el punto de vista del comportamiento de la cuenta corriente, el crecimiento sostenido del stock de IED puede llegar a afectar la sustentabilidad de las cuentas externas y de un proceso de expansión de la inversión. Durante la última década, el impacto positivo de la reducción de intereses por el pago 
de la deuda externa se vio contrarrestado por un aumento dramático en el déficit de utilidades y dividendos, vinculados con el proceso de extranjerización (Bekerman y Vázquez,2016).

- Intereses de las casas matrices: las filiales de firmas trasnacionales responden a los intereses de sus casas matrices que, por lo general, distan de reflejar las necesidades locales, y se dirigen hacia sectores o actividades que pueden limitar el desarrollo del país receptor. En las palabras de Ferrer (2014) “es dificil avanzar hacia la frontera tecnológica, en un sistema hegemónico de filiales, en el cual la actividad local no incluye la innovación de frontera".

- Trabas al desarrollo de las industrias nacionales: cuando las decisiones de compra de insumos son tomadas a nivel global, las filiales de las grandes empresas extranjeras tienden a no generar eslabones productivos hacia atrás, sino que los importan. Esto hace que, por lo general, sean mucho más importadoras que el resto de las grandes empresas locales, lo que limita la inversión local en esos rubros. En consecuencia, no se producen derrames de conocimientos directos ni indirectos (tecnológicos y de proveedores) hacia las actividades locales, lo que trae aparejado un limitante hacia la innovación local.

- Bienes monopólicos: gran parte de las trasnacionales se insertan en los mercados locales con bienes monopólicos, con elevadas barreras (fisicas e institucionales) a la entrada para otros productores que no cuentan con la escala o tecnología suficiente para contrarrestar el poder de mercado de dichas empresas.

- Diferencial de precios: En países como Argentina los sectores de bienes intermedios son los que mayor grado de concentración y poder de mercado presentan, lo que impacta en los precios de los insumos para el resto de las empresas. Esta situación se ve agravada en períodos de 
inestabilidad macroeconómica o de alta inflación en donde pueden imponer un vector de precios relativos que aumente sus beneficios.

\section{II.A.2. Heterogeneidad estructural}

La economía argentina sufre la coexistencia de sectores económicos que presentan una fuerte diferenciación en los niveles de productividad, lo que se traduce en la existencia de una estructura social dispar y nos remite a un concepto clave de la teoría latinoamericana del desarrollo: el de heterogeneidad estructural (Pinto, 1976).

Las políticas de desregulación económica, liberalización financiera y apertura comercial que tuvieron lugar a partir de mediados de los años noventas -acompañadas por un tipo de cambio fijo en paridad con el dólar entre 1992 y 2002- han ampliado los diferenciales tecnológicos entre ramas de actividad y en el interior de cada una de ellas. Al mismo tiempo, generaron excedentes relativos de población "sobrante" provocan una mayor segmentación de los mercados de trabajo donde cobra fuerza el fenómeno de la informalidad (Cimoli, Primi y Pugno, 2006).

Esta realidad, que no resulta fácil de revertir, junto a una especialización exportadora sesgada a favor de la producción primaria, puede determinar que el tipo de cambio que asegura buenos niveles de competitividad para los productos primarios no sea el indicado para permitir el desarrollo de ventajas comparativas dinámicas en otros sectores productivos y puede por lo tanto limitar el nivel de inversión en estos sectores. ${ }^{4}$ Por otra parte, la existencia de altos niveles de informalidad, a la vez que perjudica a la población involucrada (acceso a jubilaciones, a obra social, etc), le impide al Estado recaudar una parte significativa de recursos para financiarse, lo que limita su capacidad para proveer los incentivos y bienes públicos necesarios para promover la inversión y el crecimiento. Esto genera un círculo vicioso de estancamiento y de mayores niveles de presión tributaria con respecto a aquellos países que muestran menores grados de informalidad. Finalmente, la señalada segmentación de los mercados de trabajo, con la consiguiente desigualdad de ingresos y la existencia de elevados niveles de pobreza, limita la expansión del mercado interno acotando así los incentivos a la inversión y, como veremos en el punto siguiente, actúan como un obstáculo para la expansión del capital humano. ${ }^{5}$ Por eso, cuanto menor es la desigualdad en un país, mayor es la probabilidad de que sea innovador.

El impacto negativo de la desigualdad en la distribución del ingreso sobre la inversión puede ser observado en el Gráfico $\mathrm{N}^{\circ} 5$, que muestra una relación casi inversa entre la participación del 10\% de la población con mayores ingresos y la tasa de inversión. 


\section{Gráfico No. 5}

Evolución de la Tasa de Inversión y la Particiapación en el ingreso del 10\% de la población con mayores ingresos - en porcentajes -

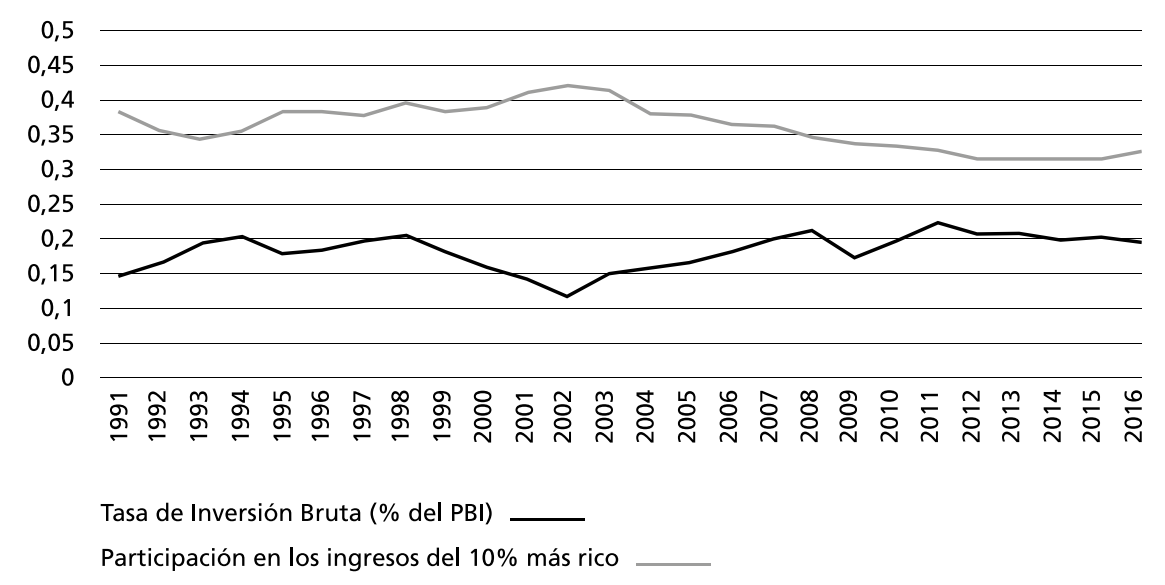

Fuente: elaboración propia en base a datos del INDEC y el Banco Muncial

\section{II.A.3. Subinversión en Capital Humano}

Una de las características sobresalientes de inversión en capital humano es su capacidad para generar beneficios a largo plazo, tanto para la persona (mejora de ingresos y satisfacciones personales) como para el resto de la sociedad, a partir del desarrollo de externalidades positivas por el beneficio social resultante del aumento del conocimiento y la productividad (Amsden 1994).

Por diversas circunstancias, el libre mercado tiende a propiciar una situación crónica de subinversión privada en capital humano. Las empresas son reacias a invertir en el desarrollo de competencias laborales debido al riesgo de perder al personal capacitado o aumentar el costo de retenerlo. Esto tiene lugar especialmente en el caso de las pequeñas y medianas empresas. Pero, además de desalentar la generación de un beneficio social, esta realidad excluye de las oportunidades formativas a los grupos sociales de menores recursos. Particularmente es en las situaciones de desempleo, pobreza y marginación donde la subinversión en educación y desarrollo del capital humano ocurren con mayor frecuencia. Esta realidad adquiere un peso especial en el caso de la Argentina, dada la existencia de altos niveles de pobreza e informalidad. 


\section{II.A.4. Mercado financiero concentrado e incompleto}

Otro de los motivos que impacta negativamente en la inversión del empresariado local se encuentra en la dificultad para financiar sus proyectos. Tal como señala Fanelli (2004) "la intermediación financiera es un insumo clave del crecimiento por sus efectos sobre la asignación y la acumulación de capital". Los altos grados de concentración y otras fallas de mercado determinan que en los países latinoamericanos sean las grandes compañías las que se ven más beneficiadas por el acceso al crédito, ya que cuentan con mayores garantías y un alto grado de protección legal e institucional. En el caso de la Argentina, los datos del Banco Mundial (2010) señalan que el crédito interno al sector privado alcanza al 13,5 \% del PIB, contra el 68\% que presenta en Brasil. En este marco, se genera que en las empresas argentinas el financiamiento a través de proveedores sea superior al promedio tanto de América Latina como al del conjunto de los países. Esto lleva a que el $43 \%$ de las empresas encuentren el acceso al financiamiento como uno de los principales limitantes al crecimiento (Gráfico $\left.N^{\circ} 6\right)$.

\section{Gráfico $\mathbf{N}^{\circ} 6$}

Financiamiento empresarial - en porcentaje del total de empresas manufactureras a 2010 -

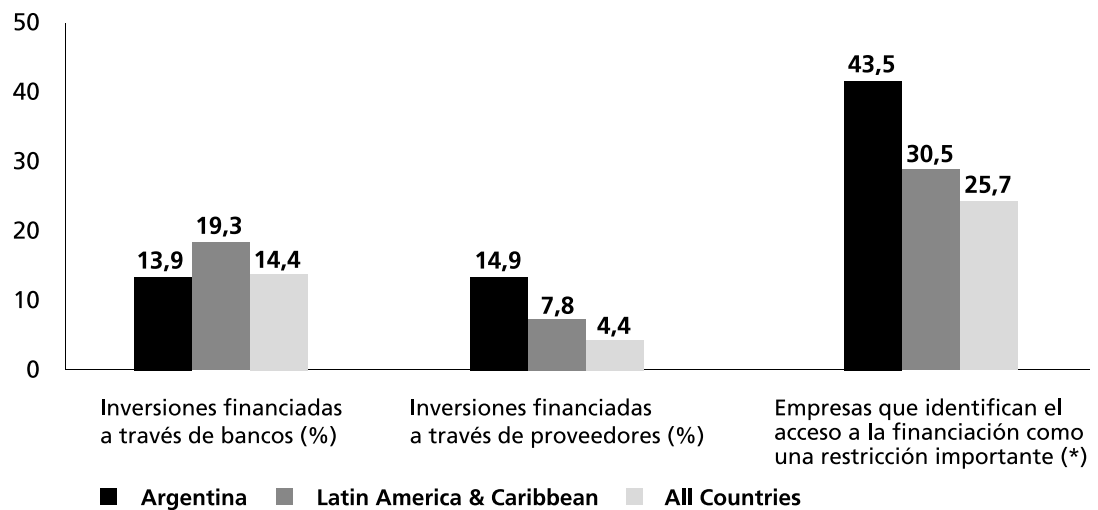

^elaboración propia en base a Enterprise Surveys - The World Bank, 2010.

En esta línea cabe resaltar que son las PyMEs las más afectadas por dicha problemática, ya que son las que recurren a financiarse en mayor medida a través de proveedores, en detrimento del sistema bancario (Gráficos $\mathrm{N}^{\mathrm{OS}} 6$ y 7). 


\section{Gráfico $\mathbf{N}^{\circ} 7$}

Inversiones financiadas a través de proveedores - en porcentajes -

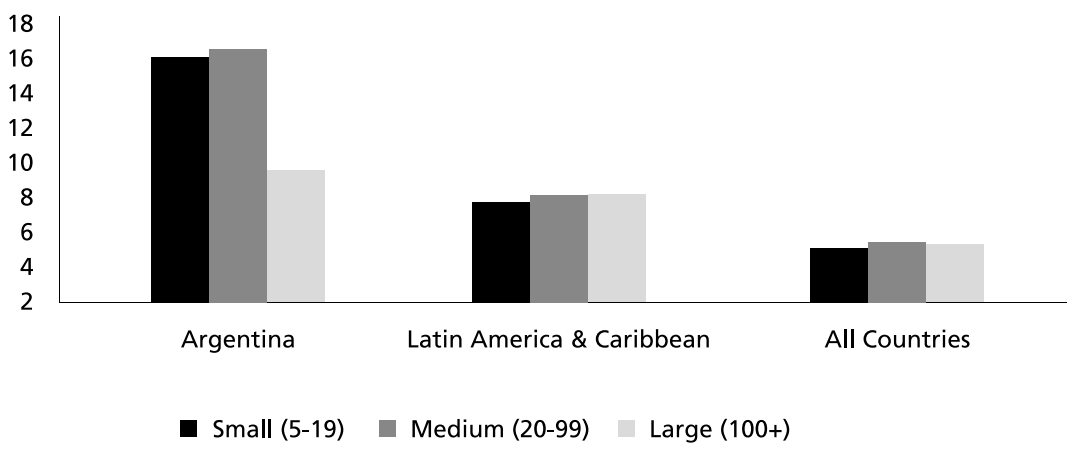

^elaboración propia en base a Enterprise Surveys - The World Bank, 2010.

Otra fuente de financiamiento para las empresas, además del sistema bancario, es el mercado de capitales. No obstante, a nivel local continúa siendo muy pequeño y fuertemente concentrado. Por otro lado, cabe resaltar que en contextos inestables los títulos de deuda pueden llegar a rendir, en promedio, más que las acciones, lo que impacta negativamente en el financiamiento empresario. En esta línea, en contextos macroeconómicos inestables, con alto endeudamiento del sector público, la capacidad de inversión del sector privado se ve reducida. Es que en la medida en que se incrementa la deuda pública (a través de emisión de bonos), se limitan las posibilidades de endeudamiento del sector privado, lo que tiene un efecto negativo sobre la inversión (crowding out).

\section{II.B Limitantes derivadas de las políticas económicas}

Las políticas económicas, en cuanto dispositivos que formulan los gobiernos para conducir la economía de los países, se basan en la implementación de ciertas herramientas orientadas a alcanzar resultados económicos específicos que pueden ser de corto o largo plazo.

Lo más frecuente es encontrar enfoques limitados a la implementación de políticas de corto plazo, normalmente asociadas a medidas coyunturales como la reducción de la inflación. Sin embargo, se requieren medidas de mediano-largo plazo para lograr objetivos que se orienten a afectar la estructura económica de un país, como, por ejemplo, incentivar el desarrollo de sectores específicos de la economía, mejorar la distribución del ingreso, etc. Pero no siempre es posible realizar una separación temporal sobre el impacto de las políticas, dado que las medidas de corto plazo pueden tener un efecto en el largo y viceversa.

Es indudable que ciertas características que presenta la estructura económica, como las analizadas en la sección anterior, pueden condicionar las posibilidades de generar 
estrategias de transformación productiva, limitando las posibilidades de innovación e incremento de productividad. Pero, en ese contexto, esa estructura puede ser modificada gradualmente a través de la correcta implementación de políticas públicas, especialmente de aquellas de carácter estructural. Una incorrecta aplicación de dichas políticas, no solo puede desincentivar a las inversiones productivas, como se analizará a continuación, sino también imposibilitar una transformación de la estructura productiva que promueva un sendero de crecimiento sustentable.

\section{II.B.1. A Nivel Macroeconómico: Impacto de la inestabilidad}

La volatilidad macroeconómica, así como los cambios frecuentes en las reglas de juego y en los precios relativos, y la consiguiente incertidumbre que generan, impactan negativamente en los comportamientos de inversión e innovación por parte del empresariado. "Cuando el entorno macroeconómico es volátil es muy difícil calcular el valor real que tendrá un contrato en el futuro" (Fanelli, 2004). Esta realidad produce conductas de "preferencias por la flexibilidad", alejadas de una visión de mediano y largo plazo, que lleva a postergar proyectos de inversión de índole innovadora y, por lo tanto, impide la generación de transformaciones positivas en la estructura productiva (Katz-Bernat, 2013).

Por otro lado, en la medida en que las políticas de estabilización se vuelven cada vez más frecuentes (debido a los contextos de incertidumbre), son tomadas con menores grados de credibilidad y mayores niveles de cautela por parte de los agentes para adaptar sus conductas ante esas nuevas políticas. Asimismo, en contextos de alta incertidumbre, los riesgos de los proyectos de innovación y creación de tecnologías de punta son exponencialmente mayores que en economías más estables, por lo que la posibilidad de desarrollar un empresariado innovador se torna más dificil. La inestabilidad termina favoreciendo la inversión especulativa en detrimento de la productiva. Es por eso que aquellos países que tienen la capacidad de adaptar mejor las políticas macroeconómicas ante shocks exógenos, son los que tienen mayores posibilidades de asegurar un crecimiento sostenido.

II.B.2 A nivel meso-económico:falla del Estado en la generación de incentivos y en la provisión de bienes públicos

Las políticas mesoeconómicas cobran una gran importancia para el desarrollo económico, ya que brindan el contexto donde se pueden crear las condiciones básicas y necesarias que estimulen, no sólo la inversión e innovación, sino también la propia generación de un sector empresarial dinámico. Este sector no deja de ser determinado, finalmente, por una construcción política (Ferrer, 1956). Además, no solo es necesario crear los incentivos correctos, sino que los mismos deben ser temporalmente acotados y atados a metas específicas. Caso contrario se pueden generar rentas extraordinarias a determinadas actividades o industrias, desincentivando en última instancia a la innova- 
ción productiva. Esto fue señalado por Schumpeter (1957) quien si bien aprobaba el comportamiento de los monopolios innovadores, que podían derramar nuevas tecnologías al resto de la economía, veía un riesgo potencial en aquellos que no innovaban ni eran de carácter transitorio.

Por otro lado, se vuelve esencial corregir las fallas en la provisión de bienes públicos de modo de asegurar el desarrollo de infraestructuras críticas (físicas, tecnológicas, intangibles). La mala calidad y limitada oferta de infraestructura constituye una fuente importante de altos costos para todos los productores y consumidores en la economía. Su eficiente provisión es necesaria debido a que, al mismo tiempo de poder impulsar la demanda de empleo, incrementa la productividad y genera espacios para invertir e innovar. Por otro lado, todo deterioro en el sistema educativo o el desfinanciamiento del sistema de ciencia y técnica pueden considerarse como factores explicativos de un declinante desempeño innovador en el largo plazo.

Otro de los limitantes mesoeconómicos para lograr un sendero de inversiones estables y eficientes es la falla en la coordinación, tanto público-privada, como público-pública. Con respecto a la coordinación público-privada, se generan distorsiones cuando los privados ven al Estado como un problema en cuanto a la imposición de trabas, al tiempo que los funcionarios públicos desconfian de las conductas rentistas de los actores privados. Ello así porque la falta de conocimiento, información y confianza limita un correcto diálogo y coordinación entre ambos sectores. Por su parte la falla en la coordinación público-pública, puede llevar a que un determinado proyecto no pueda ser realizado. Un ejemplo clásico en este tipo de falla es la promoción de un nuevo lugar turístico, para lo que se requiere la colaboración y coordinación de los diferentes ministerios para, por ejemplo, pavimentar o crear nuevos caminos/rutas, mejorar la hotelería del lugar, aumentar la frecuencia en los vuelos, que haya personal calificado para atender a los turistas, entre otros. Por dicho motivo, es necesario asegurar la coordinación e intervención de distintos organismos públicos.

Con una estructura productiva desequilibrada y sin políticas eficientes y duraderas que puedan modificar la misma, es difícil generar el contexto para que surjan inversiones que aumenten la productividad de la economía y que estimulen la innovación. Por ello en la siguiente sección analizaremos una serie de políticas para lograr un sendero de crecimiento a largo plazo.

\section{Políticas de Desarrollo frente a los limitantes a la inversión e innovación}

De acuerdo con las limitantes expuestas en la sección anterior, abordaremos aquí un conjunto de lineamientos de Políticas de Desarrollo Productivo (PDP) que permitan incentivar la inversión productiva e innovaciones. Entendemos que estas políticas 
actúan sobre la composición sectorial de la producción y regulan tanto las relaciones inter-empresariales como intersectoriales, donde se define la generación, apropiación y distribución de la renta (Porta, 2012). Incluyen un amplio espectro: tanto las que afectan el desarrollo de diversos tipos de industria naciente, como a las políticas comerciales, de ciencia y tecnología, de contratación pública, las que afectan a las inversiones extranjeras directas, y la asignación de recursos financieros, entre otras. En términos generales dichas políticas pueden ser clasificadas como de carácter horizontal o vertical.

\section{III.A. Políticas horizontales}

Los niveles de eficiencia con que se utilizan los recursos productivos y, consecuentemente, los niveles de productividad de la economía, dependen tanto de las habilidades y capacidades propias de cada una de las unidades microeconómicas (firmas) como de los factores externos a las mismas y que constituyen el entorno en el que éstas se desenvuelven. Dicho contexto puede ser beneficiado por la implementación de políticas óptimas que contribuyan a resolver las fallas de mercado existentes. Esas políticas, que pueden ser enmarcadas en el nivel mesoeconómico están vinculadas a la medida en que las firmas llegan a insertarse en redes articuladas, dentro de las cuales los esfuerzos de cada empresa se ven apoyados por toda una serie de externalidades, servicios e instituciones.

Este tipo de políticas, de carácter horizontal, apunta a mejorar el entorno global e incentivan la competitividad sistémica. Las políticas incluyen y/o benefician a todas las ramas de actividad, sin seleccionar un sector o actividad "ganadora". No obstante, cabe resaltar que en la práctica el límite entre las políticas de carácter horizontal y las de carácter vertical suele ser bastante difuso, ya que en muchos casos las primeras pueden llegar a favorecer ciertas actividades sobre otras. Por dicho motivo, los responsables políticos no pueden dejar de lado los efectos asimétricos que pueden derivarse de sus intervenciones "horizontales" y deben asegurarse que las actividades que están favorecidas en última instancia son las que sufren de manera desproporcionada las imperfecciones del mercado de que se trate (Rodrik, 2008).

\section{III.B. Políticas verticales}

Este tipo de políticas son las más controversiales, dado que pueden generar un sesgo en el vector de precios relativos beneficiando a determinados sectores por sobre otros respecto a lo que hubiese ocurrido si se dejaba actuar libremente al mercado. En esta línea, que la política de desarrollo productivo sea implementada de forma vertical no implica necesariamente que se seleccionen ganadores. Según Rodrik (2008), mediante la política industrial correctamente propuesta no se pretende elegir ganadores sino, mediante ciertos estímulos, descartar perdedores. Este tipo de políticas es necesario tanto para impulsar un determinado sector considerado estratégico, como para estimular a otro que genere nuevas cadenas de valor, como por ejemplo el sector autopartista. 
La elección de sectores que pueden ser considerados como estratégicos en el marco de las PDP ha dado lugar a diferentes posturas. Desde una perspectiva neo-schumpeteriana se deben considerar aquellas "actividades industriales inductoras de cambios tecnológicos y de mejoras en el entorno económico e institucional en su conjunto, lo que condiciona la organización institucional a partir del establecimiento de un sistema nacional de innovación" (Suzigan y Furtado, 2006). Por su parte Ajzenman (2015) sugiere extender la producción hacia ramas o sectores más complejos partiendo de las ventajas comparativas disponibles por un país. En ese contexto basar la estrategia en un desarrollo productivo de carácter lineal (ej: producir soja para exportar pellets de soja), puede no ser solo poco eficiente, sino equivocado. Por eso este autor considera esencial analizar en qué sectores es estratégicamente más conveniente centrarse para lograr un incremento de la productividad que impacte en forma global a la economía. Un ejemplo claro de esto es el desarrollo de la maquinaria agrícola.

Desde la perspectiva de la CEPAL (2012) los criterios para la elección de un determinado sector deben incluir: su crecimiento potencial, el valor agregado y contenido de conocimiento, el dinamismo y elasticidad de demanda del mercado internacional para sus productos y/o servicios, su peso en el PIB, el valor de sus exportaciones y la cantidad de empleo que genera.

Finalmente, una justificación para la realización de este tipo de políticas puede vincularse con criterios de equidad a partir del objetivo de incrementar los niveles de competitividad de sectores trabajo intensivo o con una alta capacidad de demanda de proveedores existentes en el mercado interno, a los efectos de lograr un proceso de expansión económica de carácter inclusivo.

\section{C. Políticas a través de bienes públicos o de intervención del mercado}

Además de la clasificación de las PDP entre horizontales y verticales, la intervención del Estado se puede dar a través de instrumentos que apuntan a incidir sobre el comportamiento de los sujetos económicos a través de la provisión de bienes públicos o a través de la intervención directa en el sistema de precios, vía incentivos o estímulos. Es decir que en las políticas realizadas a través de bienes públicos es el Estado el encargado de implementar medidas orientadas a aumentar la competitividad sin afectar el sistema de precios: desarrollo de infraestructuras, promoción del sistema científico tecnológico, protección de los derechos de propiedad, entre otros. En cambio, las políticas de intervención de mercado actúan directamente sobre los incentivos de los actores privados para influir sobre su conducta, accionando sobre el mecanismo de precios, ya sea otorgando los subsidios, exoneraciones fiscales o a través de aranceles y retenciones. 


\section{D. Interacción de políticas}

En función de las dos clasificaciones señaladas más arriba recurriremos a un esquema que combina, a partir de la creación de cuatro cuadrantes, las políticas horizontales y verticales -incluidas en el eje horizontal- con las políticas de intervención y de bienes públicos -en el eje vertical-. A partir de este esquema, que sigue al desarrollado por Crespi, Fernandez Arias y Stein, (2014), se presenta un conjunto de instrumentos que pueden verse en en el Cuadro $\mathrm{N}^{\circ} 2$.

\section{Cuadro $\mathrm{N}^{\circ} 2$}

Instrumentos de Políticas resultantes de la interacción entre los diversos tipos de Políticas Productivas

\begin{tabular}{|l|l|l|}
\hline Columna1 & Políticas Horizontales & Políticas Verticales \\
\hline Bienes públicos & $\begin{array}{l}\text { - Regulación del sector financiero } \\
\text { - Políticas orientadas a la IED } \\
\text { - Política de defensa a la competencia } \\
\text { - Desarrollo de instituciones sólidas y una coordinación eficaz entre } \\
\text { las mismas y entre el sector público y el privado } \\
\text { - Plan de Infraestructura local y regional } \\
\text { - Desarrollo del Sistema Nacional de Innovación }\end{array}$ & $\begin{array}{l}\text { - Creación de carreras } \\
\text { universitarias específicas } \\
\text { - Programas de } \\
\text { indulgencia }\end{array}$ \\
\hline $\begin{array}{l}\text { Intervención de } \\
\text { mercado }\end{array}$ & $\begin{array}{l}\text { - Parques industriales } \\
\text { - Créditos subsidiados y exoneraciones fiscales para PyMEs } \\
\text { y actividades innovadoras } \\
\text { - Ampliación de fondos para investigación, ciencia y tecnología }\end{array}$ & $\begin{array}{c}\text { - Beneficios financieros y } \\
\text { exoneraciones fiscales } \\
\text { para sectores estratégicos }\end{array}$ \\
\hline
\end{tabular}

El primer cuadrante que corresponde a políticas horizontales realizadas a través de la provisión de bienes públicos, es de los que generan mayor aceptación pero que no siempre son implementadas eficazmente. En este cuadrante se incluyen, entre otros, los esfuerzos para mejorar la calidad educativa o de bienes públicos que permiten reducir los costos de transacción y el respeto de los derechos de propiedad, entre otros. Es decir que este cuadrante incluye los requisitos básicos o necesarios para enmarcar con éxito a las políticas de desarrollo productivo.

Entre otros instrumentos de políticas horizontales implementadas a través de bienes públicos podemos señalar los siguientes:

Regulación del sector financiero. Si bien la regulación del sector financiero es una intervención vertical, ya que atañe a un determinado sector, la realidad es que termina generando un impacto en una innumerable cantidad de actividades. Dicha regulación debería incluir a las líneas de crédito subsidiadas para inversiones de capital, de I+D y de formación de capital humano, fundamentalmente enfocada para las PyMEs. Asimismo, la regulación debería abarcar la expansión y modificación del mercado de capitales como fuente alternativa de financiamiento de las empresas nacionales. 
Políticas orientadas a la IED. La inversión extranjera directa puede aumentar la productividad de un país, pero si no es eficientemente regulada puede tornarse un factor limitante al desarrollo. Por ello, son necesarias políticas que la regulen, para que la misma impacte positivamente en la estructura local y genere eslabones productivos tanto hacia atrás como hacia delante, posibilitando un derrame de conocimiento que pueda ser apropiado por las empresas y actores locales. Es decir, se requieren políticas para que las empresas transnacionales generen incorporación y transferencia tecnológica al entramado nacional, que incrementen empleo local y expandan las exportaciones, al tiempo que generen eslabonamientos con empresas locales. No obstante, este tipo de políticas no son fáciles de realizar en economías con un mercado pequeño o con poco poder de negociación, como es el caso de la mayor parte de las economías latinoamericanas.

Política de defensa a la competencia. Si bien en Argentina existe una ley para la defensa de la competencia (Ley 25.156), aún el país está atrasado en normativas con respecto al resto de la región. Un ejemplo de esto es la falta de soluciones efectivas a los cárteles nocivos, entendiéndose a los mismos como aquellos que limitan la competencia y ejercen poder en la "formación de precios, fijación de cuotas de mercado o volúmenes de producción, concertación de posturas en licitaciones, y limitación a la investigación y desarrollo" (Malis et al. 2003).

Desarrollar instituciones sólidas y una coordinación eficaz entre las mismas. Es necesario contar con marcos regulatorios estables y políticas públicas duraderas, que no dependan de los cambios de gobierno y/o de las reglas de juego imperantes. Asimismo, es fundamental desarrollar instituciones que muestren una consolidación y fortalecimiento en el tiempo, junto con un proceso de coordinación eficaz entre las acciones de las mismas. Una de las alternativas para incrementar la coordinación público-pública, expresada por Hausmann (2016), consiste en destinar un porcentaje del presupuesto a un fondo común a ser demandado por un determinado ministerio, pero ejecutado por otro, así como consignar un determinado momento para el debate de las ideas, propuestas y objetivos de los representantes de los distintos ministerios. Es asimismo importante consolidar una eficiente coordinación público-privada como motor para incentivar la generación de nuevas inversiones y añadir valor al conjunto de la sociedad. Existen numerosas experiencias de realización de "mesas ejecutivas" sectoriales, como las desarrolladas por el gobierno peruano, en las que los representantes públicos y privados se reúnen con el propósito de identificar y remover las barreras específicas al crecimiento de cada sector. ${ }^{6}$

Plan de infraestructura local y regional. Este tipo de política tiene un doble objetivo, por un lado, beneficiar a las regiones y empresas involucradas y, por el otro, generar empleo, vía construcción de infraestructuras tales como caminos, escuelas, redes ferroviarias o energéticas, que generan un beneficio tanto para las empresas como para la sociedad en su conjunto. Asimismo, este tipo de política tiene por objetivo fortalecer los lazos comerciales y políticos de la región. 
Desarrollo del Sistema Nacional de Innovación (SNI). En Argentina hay investigadores y científicos sumamente calificados y universidades prestigiosas a nivel internacional. El problema está en la falta de interacción y articulación del conjunto de los actores y su interconexión con el sector público. Es por ello que se necesita desarrollar el SNI. Argentina no sólo cuenta actualmente con uno de los menores gastos en I+D (en torno al 0,4\% del PIB), tanto a nivel mundial como regional, sino que, además, dichos gastos están realizados en su gran mayoría con financiamiento del sector público. Es decir que es escasa la participación de las empresas en actividades de I+D. Por lo que, además de incentivar esta inversión por parte del sector privado, es esencial implementar un sistema de difusión de las innovaciones en ciencia básica que son realizadas en el país, y que, por diversas razones, quedan en el plano puramente académico. La performance innovativa de un país depende, entonces, de cómo estos diferentes actores se relacionan entre sí como elementos de un sistema colectivo de creación y uso de conocimiento (López, 2006).

El segundo cuadrante incluye la provisión de bienes públicos que generan beneficios para un sector específico. Dentro de los diversos ejemplos podemos mencionar: los controles fitosanitarios, la promoción pública de investigaciones para crear nuevas variedades de cultivos, el fomento al turismo, la coordinación logística para facilitar y estimular la exportación de ciertos productos, entre otros. Si bien se trata de políticas de carácter vertical no puede dejar de tenerse en cuenta sus posibles impactos para el conjunto del entramado socioeconómico. En este sentido, la creación de ciertas carreras universitarias puede ser considerada como un bien público, vinculado con la educación, y de carácter vertical por el hecho de estar dirigido hacia un sector específico. Tal es el caso, por ejemplo, de la carrera de Ingeniería Nuclear en el Instituto Balseiro que, a pesar de estar vinculada a un sector específico, puede generar importantes derrames hacia otros sectores, al tiempo de estimular el trabajo calificado.

Programas de indulgencia, como herramienta para regular cárteles y disminuir los niveles de concentración. Estos programas, que fueron incorporados por algunos países de la región, como Brasil (desde el 2000) y Chile (2009), apuntan a conocer el funcionamiento de los cárteles existentes, usualmente desconocidos y de detección dificil. Para lograrlo, el programa de indulgencia reduce (o elimina) las sanciones económicas al primer solicitante, teniendo éste que facilitar toda la información pertinente y concluir la actividad en el cártel. No obstante, para que el programa funcione, se requiere que la ley de competencia y sanción para los cárteles sea lo suficientemente creíble, eficiente y con altos costos para aquellos que la incumplen. Actualmente en Argentina no hay programas de indulgencia, por lo que se debería modificar o ampliar la ley actual de competencia, con el fin de incluirlos y poseer una herramienta más para fortalecer el impacto de dicha política. 
El tercer cuadrante, que involucra a políticas horizontales ligadas a intervenciones de mercado, apunta a promover determinadas actividades que pueden estar afectadas por la existencia de fallas de mercado. Incluye distintos mecanismos tales como desgravaciones fiscales y créditos subsidiados, entre otros. La clave consiste en identificar cuál es la falla de mercado que se pretende resolver, dado que su existencia genera una diferencia entre el beneficio social y el beneficio privado percibido por los agentes del mercado que pueden desarrollar estas actividades.

Parques Industriales. Se trata de espacios diseñados para la localización de industrias y servicios afines a las mismas, que cuentan con la infraestructura necesaria y espacios comunes a las diversas compañías. Estos predios pueden generar economías de aglomeración, favorecer el desarrollo de nuevas PyMEs, estimular el desarrollo de eslabones productivos, al tiempo que pueden ser una fuente de empleo genuino y de aumento de competitividad de las empresas allí localizadas. Los situamos en el tercer cuadrante, ya que por lo general los gobiernos otorgan incentivos fiscales a las empresas que se instalen en los mismos. Los parques industriales han tenido un notable éxito en algunos países latinoamericanos, como son los ejemplos de São Paulo en Brasil, Parque Logístico Nacional del Tolima en Colombia, Rafaela (provincia de Santa Fe) en Argentina, Parque Industrial Coronel en Chile, Canelones en Uruguay, entre otros.

Créditos subsidiados y exoneraciones fiscales para PyMEs y actividades innovadoras. Estas herramientas deben estar orientadas tanto hacia la incorporación de bienes de capital como para la capacitación del personal y a la innovación, con el fin de incrementar la productividad y eficiencia. "En numerosas actividades, la revolución tecnológica contemporánea ha eliminado las economías de escala, habilitando a las PyMEs a operar con los conocimientos de frontera" (Ferrer, 2014). Por otro lado, estas empresas juegan un rol fundamental en la economía argentina, en la demanda de empleo, en la competitividad de la estructura económica, y en la generación de innovaciones productivas y tecnológicas.

Subsidios para inversión en capital humano. Una herramienta posible en este campo es la asignación de fondos públicos para la formación profesional, tanto del lado de la oferta como de la demanda. Con respecto a los subsidios a la oferta, deberían destinarse a instituciones de formación profesional reconocidas y a proveedores de servicios formativos, ya sean privados o públicos. Una alternativa a la financiación directa es la financiación pública de centros de formación dirigidos por las empresas, de manera individual o colectiva. Este esquema favorece una mayor articulación de la oferta formativa con las necesidades reales en el sector privado. Por el lado de la demanda, los subsidios pueden ser otorgados a los usuarios de los servicios formativos, ya sean trabajadores o empresas. (Martínez et al.2007)

En el cuarto cuadrante se ubican las PDP más polémicas, aquellas que intervienen en el mercado (subsidios, exoneraciones fiscales) y que se aplican en forma vertical, es decir 
seleccionando a un sector en particular. Una intervención de carácter vertical puede ser justificada en situaciones que contribuyen a solucionar problemas de coordinación en sectores con ventajas competitivas latentes o a estimular el desarrollo de encadenamientos productivos tanto hacia atrás como hacia delante. Aquí es importante destacar, con base en la experiencia de algunos países asiáticos, que las políticas de carácter selectivo deben ser transitorias y con metas específicas, de modo de evitar conductas captadoras de renta (rent-seeking).

Beneficios financieros y exoneraciones fiscales para sectores estratégicos: Un claro ejemplo es el sector del software en Argentina, implementado a través de la ley de promoción de esa industria (ley 25.922), reglamentada en 2004, en la cual se enumeran los beneficios para dicho sector, tales como las exenciones impositivas, beneficios crediticios y subsidios. Esto posibilitó no solo un crecimiento significativo de dicho sector, sino que contribuyó, también, a diversificar la canasta exportadora, al tiempo que incrementó la demanda de empleo calificado y de nuevos actores empresariales.

\section{Conclusiones}

A partir de los condicionantes internos y externos que enfrenta actualmente la región para lograr un proceso de transformación estructural que permita reducir la concentración de la matriz exportadora en productos primarios y establezca las bases para un crecimiento sostenido e inclusivo a largo plazo, este trabajo ha enfatizado la necesidad de retomar el rol de las políticas productivas, considerando a las mismas como un instrumento clave para sortear los obstáculos que limitan la inversión productiva y la innovación. En el plano internacional prevalece, por un lado, una profundización en el proceso de globalización económica, que no ha sido acompañada por un avance similar en las regulaciones de la OMC y, por el otro, el auge de nuevas posturas proteccionistas ante lo que parece presentarse como una crisis del proceso de globalización, crisis que requiere una mirada muy atenta de las economías latinoamericanas para poder responder, frente a la misma, de la manera más eficaz. Asimismo, la desaceleración de la economía china ha generado señales de alerta a nivel global y regional.

En el plano nacional, observamos que aparecen serias limitaciones en el comportamiento de la inversión, como paso necesario para un proceso de innovación que posibilite la transformación de la estructura productiva. El trabajo señala dos conjuntos de limitantes fundamentales: aquellos derivados de las características que presenta la estructura productiva y los resultantes de políticas económicas inefectivas. Al tiempo que se toma en cuenta la relación existente entre ambos aspectos. Es que la característica que presenta la estructura económica condiciona los resultados de las políticas, a la vez que la implementación de ciertas políticas puede modificar dicha estructura, lo cual puede ser un determinante para avanzar en un proceso de crecimiento sostenido. 
A partir de allí se presenta un conjunto de instrumentos resultantes de la interacción entre políticas horizontales y verticales con los orientados a la provisión de bienes públicos o a la intervención directa, vía incentivos o estímulos vía subsidios o exoneraciones fiscales. Asimismo se señala la importancia de que dichas políticas puedan ser sostenidas a partir del desarrollo de políticas macroeconómicas sustentables, reconociendo el impacto que cada una de estas esferas genera sobre la otra. Ello así porque los resultados de las políticas productivas no serán los mismos bajo un contexto macroeconómico inestable, a la vez que no puede dejar de tomarse en cuenta su impacto sobre las condiciones estructurales.

En este contexto se resalta, como uno de las condiciones fundamentales, el desarrollo de instituciones sólidas que permitan asegurar la efectividad del diseño e implementación de los instrumentos de política, y que aseguren su continuidad en el tiempo. En la realidad práctica, la mejora de la eficiencia dinámica demanda cambios estructurales que pueden ser lentos y costosos. Por tal motivo estos cambios deben basarse en políticas que aseguren que las inversiones y las actividades económicas puedan presentar características sustentables, y que no se basen en estructuras de incentivos transitorias o inciertas; todo lo cual requiere asegurar el desarrollo eficiente de la coordinación público-privada.

Asimismo, las condiciones que se presentan en el escenario internacional, de fuerte avance del proceso de globalización por un lado y de marcadas presiones proteccionistas de las economías centrales por el otro, generan nuevas implicancias y una necesidad de reorientación para las políticas productivas de los países de América Latina. Esto se da en un contexto de marcada debilidad del MERCOSUR y del comercio regional. Por ello se enfatiza la importancia de que, a los efectos de potenciar el impacto de las políticas de desarrollo productivo, se avance hacia un afianzamiento de las relaciones económicas regionales para fortalecer la capacidad negociadora internacional y mejorar la competitividad a través de la expansión de los mercados nacionales, lograr una integración de la infraestructura regional y avanzar hacia la creación de cadenas de valor regionales. 
${ }^{1}$ Formación Bruta de Capital Fijo (FBCF): Mide el valor de las adquisiciones de activos fijos nuevos o existentes menos las cesiones de activos fijos realizados por el sector empresarial, los gobiernos y los hogares. ${ }^{2}$ A su vez, la facturación de las doscientas firmas más grandes representaba el 43,5\% del PBI argentino en 2012 (Fuente: INDEC).

${ }^{3}$ Sólo se observa una reducción en la participación del capital extranjero en los sectores de petróleo y maquinaria. En el primer caso esto se vincula con la renacionalización de YPF en al año 2012. En el sector de maquinaria, se explica por el crecimiento del complejo ensamblador de artículos electrónicos de Tierra del Fuego, anclado en empresas nacionales

${ }^{4}$ Marcelo Diamand explicó esta situación bajo el concepto de Estructura Productiva Desequilibrada, la que puede verse agudizada en períodos de fuertes aumentos en los precios internacionales de los bienes primarios como tuvo lugar en un período reciente.

${ }^{5}$ En palabras del director de Oxfam en Asia: "la desigualdad limita la inversión productiva y la capacidad de consumo de la sociedad, lo que pone en peligro el crecimiento a largo plazo" http://www.laprensagrafica. com/2015/01/20/oxfam-alerta-de-que-la-desigualdad-amenaza-el-milagro-economico-en-asia)

${ }^{6}$ Ver www.adexperu.org.pe/prensa/notas-de-prensa/item/934-piero-ghezzi-sector-privado-debe-pedir-alsiguiente-gobierno-continuar-mesas-ejecutivas 


\section{Referencias bibliográficas}

Ajzenman, N. (2015) “¿Industria diversificada o industria compleja?”. Diario La Nación, 12/10/2015.

Amsden A. (1994) "Why isn't the whole world experimenting with the East Asian model to develop? Review of the East Asian miracle”. World Development 22 (4):627-633

Bekerman, M. y D. Vázquez (2016) “Inversión extranjera directa y estructura productiva en la posconvertibilidad”. Revista Desarrollo Económico, vol. 56, N 219:207-244.

Castells, M; E. Ferreira, E. Inchauspe y M. Schorr (2014) "Bienes de capital en la posconvertibilidad: desempeño comercial externo y (des)aprovechamiento de la masa crítica existente”. Realidad Económica No 283:127-158.

CEPAL (2012) Cambio estructural para la igualdad: Una visión integrada del desarrollo. Trigésimo Cuarto Período de Sesiones de la CEPAL, San Salvador, 27 al 31 de agosto de 2012.

Cimoli, M., Primi, A. y M. Pugno (2006) "Un modelo de bajo crecimiento: La informalidad como restricción estructural”. Revista de la CEPAL No 88:89-107.

Coremberg, A. (2015). Inversión Necesaria y su Impacto en la Economía Argentina. ¿Cuánto necesitamos para crecer? Biblioteca Cámara Argentina de la Construcción.

Crespi, G., E. Fernández Arias y E. Stein, E. (2014) ¿Cómo repensar el desarrollo productivo? Políticas e instituciones sólidas para la transformación económica. Washington DC, Banco Interamericano de Desarrollo.

Dávila-Fernández, M. (2015) “Desindustrialização e o investimento em infraestrutura como instrumento conciliador de uma política industrial base no Brasil”. Revista de Economía Política, vol. 35, no 3:576-600.

Diamand, M. (1972) “La Estructura Productiva Desequilibrada Argentina y el Tipo de Cambio”. Revista Desarrollo Económico Vol. 12 N 45.

Fajnzylber F. (1988) “Competitividad Internacional. Evolución y Lecciones”. Revista de la CEPAL N 36:7-24.

Fanelli, J.M. (2004) "Desarrollo Financiero, Volatilidad e Instituciones. Reflexiones sobre la Experiencia Argentina”. Documento de trabajo de la Fundación PENT 2004-003. 
Ferrer, A. (1956) El Estado y el desarrollo económico. Editorial Raigal

Ferrer, A. (2014) “Empresario y argentino”. Diario Perfil 13/09/2014.

Foxley, A. y F. Sossdorf (2011) Making the Transition: from Middle-Income to Advanced Economies. The Carnegie Papers, Carnegie Endowment for International Peace.

Gillman, K., L. Coutinho, E.. Amadeo, E. Campbell., M. Diamand, M., F. Kindgard, J.J. Llach, I. Mahler, A. Mayoral,V. Orsi y H. Roggero (1991) Políticas públicas y privadas hacia la competitividad industrial. Colección Ciencia y Técnica. Buenos Aires, EUDEBA

Gómez Gavira, D. y G. Angerita (2014) Política Industrial: Una propuesta de política a la luz de la tensión entre fallas de mercado y fallas de gobierno. Bogotá: (FESCOL).

Hausmann, R. (2016). Overcoming the Public-Sector Coordination Problem. https://www. project-syndicate.org/commentary/improving-public-sector-coordination-by-ricardo-hausmann-2016-10?version=spanish\&barrier=accessreg

\section{Instituto Nacional de Estadísticas y Censos de la República Argentina (IN-} DEC) Varios años. http://www.indec.gob.ar/

Katz,J. y G. Bernat (2013) “Interacciones entre la macro y la micro en la postconvertibilidad: dinámica industrial y restricción externa”. Revista Desarrollo Económico 25(207-208): 383-404.

López, A. (2006) Empresarios, instituciones y desarrollo económico: el caso argentino. Trabajo preparado para la Oficina de CEPAL Buenos Aires.

Malis, I., D. Povolo y J. Pereda (2003) La lucha anti-cartel en la Argentina. OECD. http:// www.oecd.org/daf/competition/prosecutionandlawenforcement/38858052.pdf.

Martínez, E. y R. Galhardi (2007) "La formación profesional como inversión en capital humano". En Herramientas para la Transformación $N^{\circ} 33$. Centro Interamericano para el Desarrollo del Conocimiento en la Formación.

McMillan M. \& D. Rodrik (2011) Globalization, structural change and productivity growth Ginebra: WTO. Disponible en: https://www.sss.ias.edu/files/pdfs/Rodrik/Research/ Globalization_Structural\%20Change_Productivity_Growth_with_Africa_update.pdf

Pérez, C. (2012) “Una visión para América Latina: Dinamismo tecnológico e inclusión social mediante una estrategia basada en los recursos naturales”. Revista Econômica - Niterói, v.14 (2) 11.54. 
Pinto, A. (1976) Heterogeneidad estructural y modelo de desarrollo reciente de la América Latina. México, Fondo de Cultura Económica.

Porcile, J.G. y M. Cimoli, M. (2014) “Integración económica y cambio estructural en América Latina”. Revista Puente Europa.

Porta, F. (2012) "Dinámica productiva y política industrial en Argentina". Ciencias Sociales de la UNQ N²2.

Rodrik, D. (2010) “El regreso de la política industrial”. https://www.project-syndicate. $\mathrm{org} /$ commentary/the-return-of-industrial-policy?version=spanish\&barrier=accessreg

Rodrik, D. (2008) “Normalizing Industrial Policy”. Commission on Growth and Development, Working paper $\mathrm{N}^{\circ} 3$

Schorr, M. y A. Wainer (2014) "La economía argentina en la posconvertibilidad: problemas estructurales y restricción externa”. Revista Realidad Económica N² 286:137-174.

Schumpeter, J. (1957) Teoría del Desenvolvimiento Económico. México DF: Fondo de Cultura Económica.

Suzigan W. y J. Furtado (2006) "Política Industrial y Desarrollo". Revista de la CEPAL No 89:75-91.

Tyson, L. (2016) “El faltante de inversión”. https://www.project-syndicate.org/commentary/american-business-investment-slump-by-laura-tyson-2016-02?version=spani sh\&barrier $=$ accessreg

Vázquez, E. (1988) La Argentina: Cuarenta años de inflación. Buenos Aires: El Ateneo.

Fondo Monetario Internacional (2016) World Economic Outlook Database (WEO) (2016) https://www.imf.org/external/pubs/ft/weo/2016/01/weodata/index.aspx

\section{Cómo citar este artículo:}

Bekerman, Marta y Anabel González Chiara (2018) "Limitantes a la inversión productiva como obstáculo al desarrollo. El caso de la Argentina”. Revista Perspectivas de Políticas Públicas vol. 7 №14: 239-264 